Journal of Engineering and Applied Sciences 14 (Special Issue 6): 9396-9404, 2019

ISSN: $1816-949 \mathrm{X}$

(C) Medwell Journals, 2019

\title{
Secure Image Steganography using Biorthogonal Wavelet Transform
}

\author{
Enas Muzaffer Jamel \\ Department of Computer Science, College of Education for Women, University of Baghdad, \\ Baghdad, Iraq, enasm.altai@gmail.com
}

\begin{abstract}
Hiding digital information is a spectacular scientific field. It is placed under security systems. This study, presents non-blind steganography system where biorthogonal wavelet transform is applied to decompose the gray level cover image into four sub-bands. The secret image is hidden in any one sub-band of high frequencies after applying the scrambling process. The scrambling process depends on changing positions of each row and column in the secret image. So that, will give intensive security for hiding system. Analyzing the results show that bior 6.8 and rbio6.8 provide the best results and achieve good imperceptibility and robustness compared to other biorthogonal wavelet transforms. To evaluate the results, PSNR and correlation are adopted. Best results of PSNR are obtained from hiding in HH sub-bands. The correlation values are acceptable at all sub-bands. Another comparison is achieved between Haar wavelet transform and (bior6.8 and rbio6.8) which also shows the effectiveness of the biorthogonal wavelet transforms. The proposed method is robust against noise attacks.
\end{abstract}

Key words: Steganography, biorthogonal wavelet transform, orthogonal wavelet transform, scrambling, noise attacks, scrambling

\section{INTRODUCTION}

In recent years, the security systems have become very important in protecting confidential personal data (Houssein et al., 2016). Thus, increasing the importance of data transfer in a safe manner (Kasana et al., 2017). Steganography is a technique used to hide data in information security applications. The purpose of the hiding is to provide invisible communication (Houssein et al., 2016). The information is hidden in such a way that no one can notice it as hiding the information inside media such as images to carry information securely. The image is used as a data carrier cover to protect it (Reddy and Kumar, 2016). The main characteristics needed by the information security applications are imperceptibility, capacity and robustness. imperceptibility avoids any suspicion from existence of hidden secret data but the capacity indicates the amount of data that can be stored in the host image. The robustness is used to measure the vulnerability on the steganography system against attacks (Kasana et al., 2017).

There are two types of embedding the first depends on the spatial domain of the embed signal and can be accomplished by changing the least significant bits of the bytes to image pixel values. This type of embedding can be achieved either in a random way or in a sequential way. Although, this method allows the use of algorithms with high load, it is not safe enough to be exposed to statistical or visual attacks. The second type, known as frequency domain, i.e., DCT, DWT. The frequency domain is based on the coefficients of band to embedding is more resistant to penetrations (Cheddad et al., 2008).
The wavelet transform is a mathematical method by which the spatial image domain is converted to frequency domain. Compared with DCT and DFT, the wavelet transform has less intensive resource, so that, occurs less distortion in the image (Atawneh and Sumari, 2015). The low and high frequencies are extracted by passing the image through the high and low pass filters, respectively. Wavelet transform analysis deals with the signal as a composed of the detail category and the category of approximations (Houssein et al., 2016). It analyzes the signal to different scales and frequency bands and decomposes the image into different images of the sub-band High-High (HH), Low-Low (LL), Low-High (LH) and High-Low (HL) (Reddy and Kumar, 2016). The low frequency part has information of image close for the original image. The high frequency parts have information about edge components. The steganography systems usually use the high frequency components. Since, when changes in edges, the human eye is less sensitive. And as these changes are to unnoticed, the image becomes harder to penetrate compared to other methods (Jiansheng et al., 2009). The wavelets can be either orthogonal or bi-orthogonal. Bi-orthogonal has more favorite features exceed orthogonal wavelet transform. The bi-orthogonal is considered invertible transform and the reconstruction is ideal (Divya and Priya, 2013). The symmetry in filters that makes the generated wavelet functions are also symmetrical and this is not achieved in orthogonal filters (Akhtar, 2001). This study focuses on the study of the effectiveness of the biorthogonal wavelet transform 6.8 which was used in the 
hiding process. The secret image scrambled by changing the positions of each row and column (Arvind and Shankar, 2013) used bi-orthogonal wavelet transform to applied watermarking algorithm in horizontal and vertical sub bands. The embedding process was achieved based on modified the coefficients of the sub band for the watermark. The blue channel of the cover image is selected for embedding the watermark because it is more resistant to changes when compared to red and green channels. The blue channel is decomposed inton-level by using bi-orthogonal wavelet transform This method is shown to be robust against many attacks. While Nag et al. (2011) encoded the secret image by Huffman coding before hiding it. The cover image is decomposed based on Haar wavelet transform. Huffman code is divided into 3-bits blocks that led to form a decimal value ranging from $0-7$. In high frequency sub-bands, the 3 least significant bits of wavelet coefficients is changed with the 3 bits of Huffman encoded bit stream. Kumar and Muttoo (2013) applied image steganography on different orthogonal and Biorthogonal Wavelet families. The secret message was embedded based on modified LSB method. The results found that Haar wavelet is the better option for image steganography based on modified LSB method. The proposed method is shown to be non-robust to Gaussian and salt-n-pepper noise.

Biorthogonal wavelet family: Biorthogonal families consist of biorthogonal and reverse Biorthogonal wavelets. From the family of spline functions, the biorthogonal scaling functions are determined. Biorthogonal and reverse biorthogonal include [compactly supported wavelets associated with biorthogonal spline scaling functions implemented with FIR (Finite Impulse Response) filters]. For FIR filters, both symmetry and exact reconstruction are possible (Stolojescu, 2011). Biorthogonal wavelet basis differs from the orthogonal basis where the scaling and the wavelet functions do not require the same length or even numbers. Therefore, the property of quadrature mirror property is not suitable and a dual is used instead (Toufik and Mokhtar, 2012). To perform the ideal reconstruction equation, the scaling and the wavelet coefficients have to realize Eq. 1 and 2 (Fig. 1):

$$
\begin{aligned}
& \tilde{g}(\mathrm{n})=(-1)^{\mathrm{n}} \mathrm{h}(1-\mathrm{n}) \\
& \mathrm{g}(\mathrm{n})=(-1)^{\mathrm{n}} \tilde{\mathrm{h}}(1-\mathrm{n})
\end{aligned}
$$

when, there is a similarity to the analysis and synthesis filters it makes the system orthogonal (Toufik and Mokhtar, 2012). The state of orthogonality is defined as:

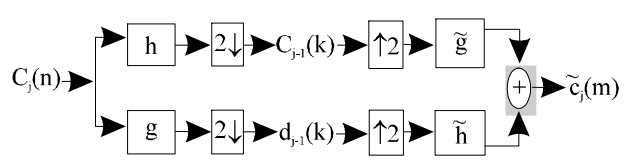

Fig. 1: Two-band analysis and synthesis filter bank
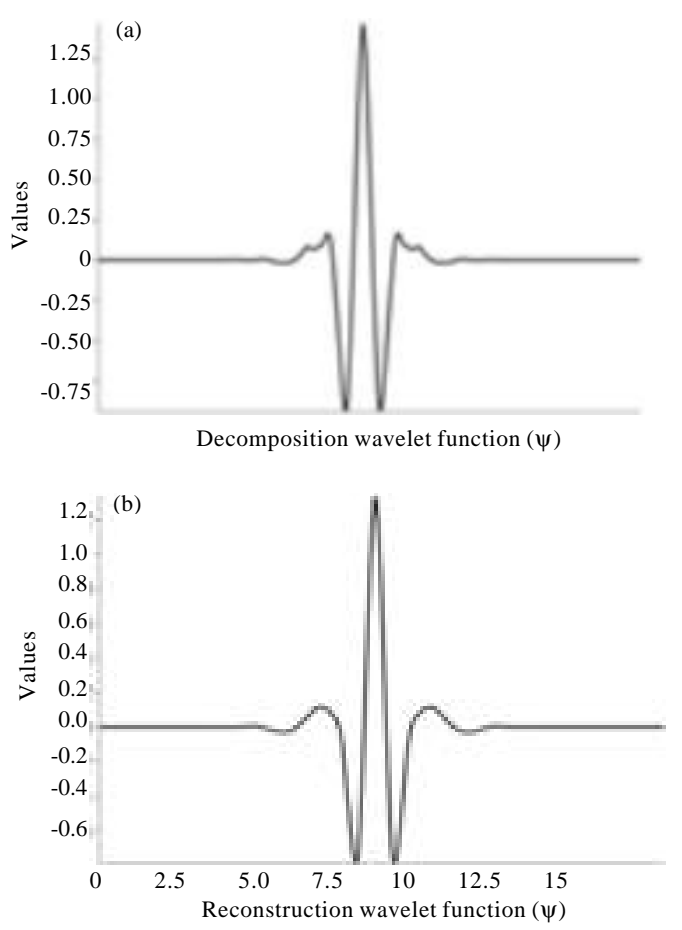

Fig. 2: a) Decomposition Biorthogonal wavelet function of 6.8 and b) Reconstruction Biorthogonal wavelet function of 6.8

$$
\sum \tilde{h}(\mathrm{n}) \mathrm{h}(\mathrm{n}+2 \mathrm{k})=\delta(\mathrm{k})
$$

In orthogonal basis, the analysis scaling coefficients are used together with their shifted versions. In biorthogonal state, the analyzing scaling coefficients does not change whereas their shifted versions are exchanged by the shifted versions of the synthesis dual filter. This means [the analysis filter is orthogonal to its synthesis dual filter]. Linear phase property is one of the most important characteristics. The filter is symmetrical in the low pass filter while it is symmetrical or asymmetrical in the high pass filter. Low or high pass filters are not considered to have similar lengths in a filter bank. The filters are either even or odd orders and the coefficients of the filters have integers or real numbers (Toufik and Mokhtar, 2012). Figure 2 shows decomposition and reconstruction Biorthogonal wavelet function of 6.8 (Wavelets, 2018). 
(a)

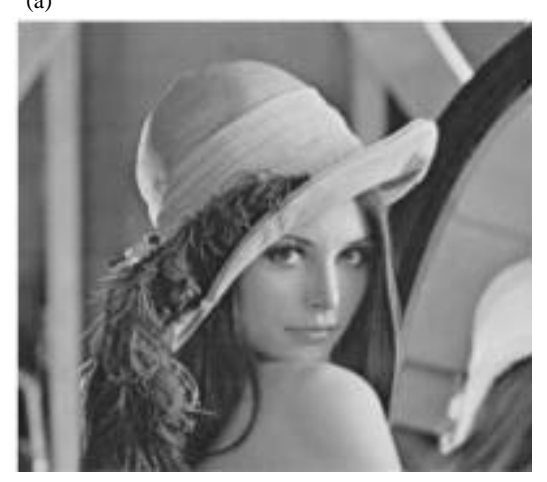

(b)

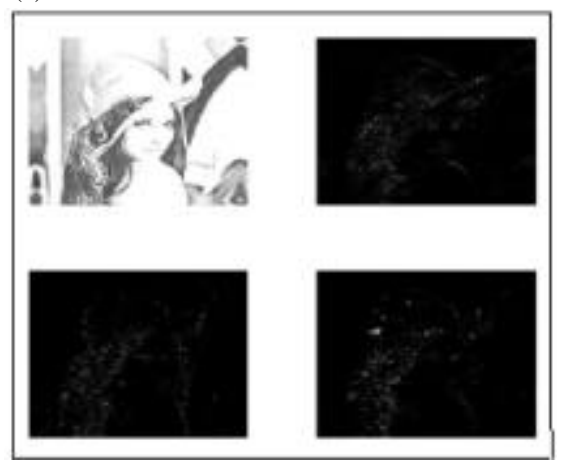

Fig. 3: a) Cover image and b) The decomposition of cover image

(a)

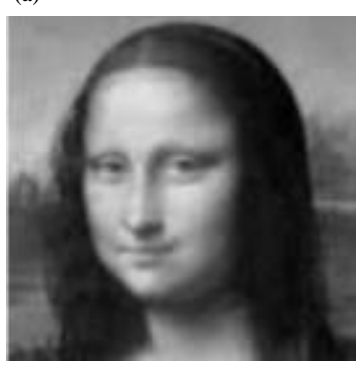

(b)

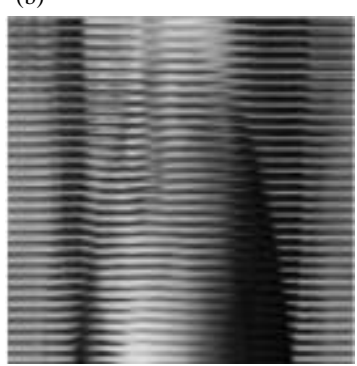

(c)

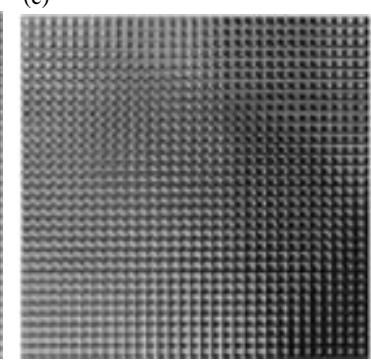

Fig. 4: a) Original secret image; b) Scrambling the rows of secret image and c) Scrambling the columns of secret image

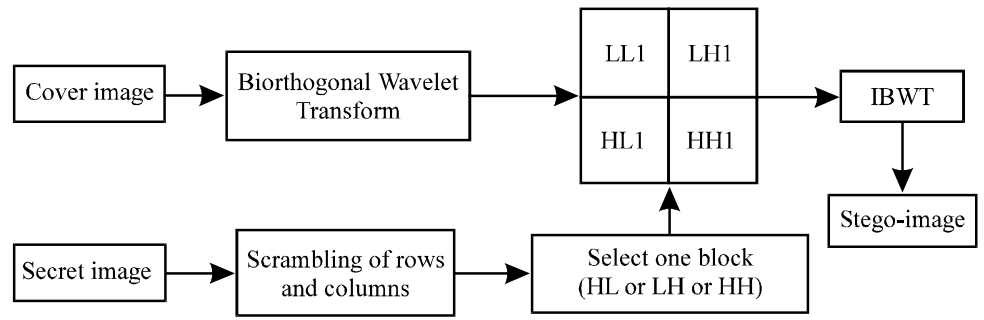

Fig. 5: The hiding process steps

\section{MATERIALS AND METHODS}

In this study, a steganography system is proposed, it depends on using Biorthogonal wavelet 6.8 , to decompose the cover image into four sub-bands. The secret image is hidden in any one of the high frequency sub-bands. Inverse Biorthogonal wavelet 6.8. is performed to obtain the stego-image.

\section{Algorithm 1; Hiding algorithm: \\ Input: Cover image, Secret image \\ Output: Stego-image}

Step 1: Apply biorthogonal wavelet transform to the cover image to get the decomposition sub-bands ( $\mathrm{LL}, \mathrm{LH}, \mathrm{HL}, \mathrm{HH}$ ) as in Fig. 3

Step 2: Scrambling of rows positions then columns positions for the secret image as in Fig. 4
Step 3: The scrambled image is Multiplied by $\bullet$ where $\cdot$ is scaling factor. (empirically $=0.05$ )

Step 4: Select any one of high sub-bands to hide secret image:

A: Secret image is hidden in $\mathrm{HH}$ sub-band

B: Secret image is hidden in $\mathrm{HL}$ sub-band

C: Secret image is hidden in LH sub-band

Step 5: Apply inverse BWT for image obtained in step 4. Figure 5 shows the block diagram of the hiding process

Extraction algorithm: The non-blind systems needs the original image to extract the secret image from the stego one (Singh and Chadha, 2013). The biorthogonal wavelet transform is performed to both the original image and the stego-image then second level of decomposition is applied to the high frequency sub-bands. At the second level. The coefficients of low-low sub-band in original 
J. Eng. Applied Sci., 14 (Special Issue 6): 9396-9404, 2019

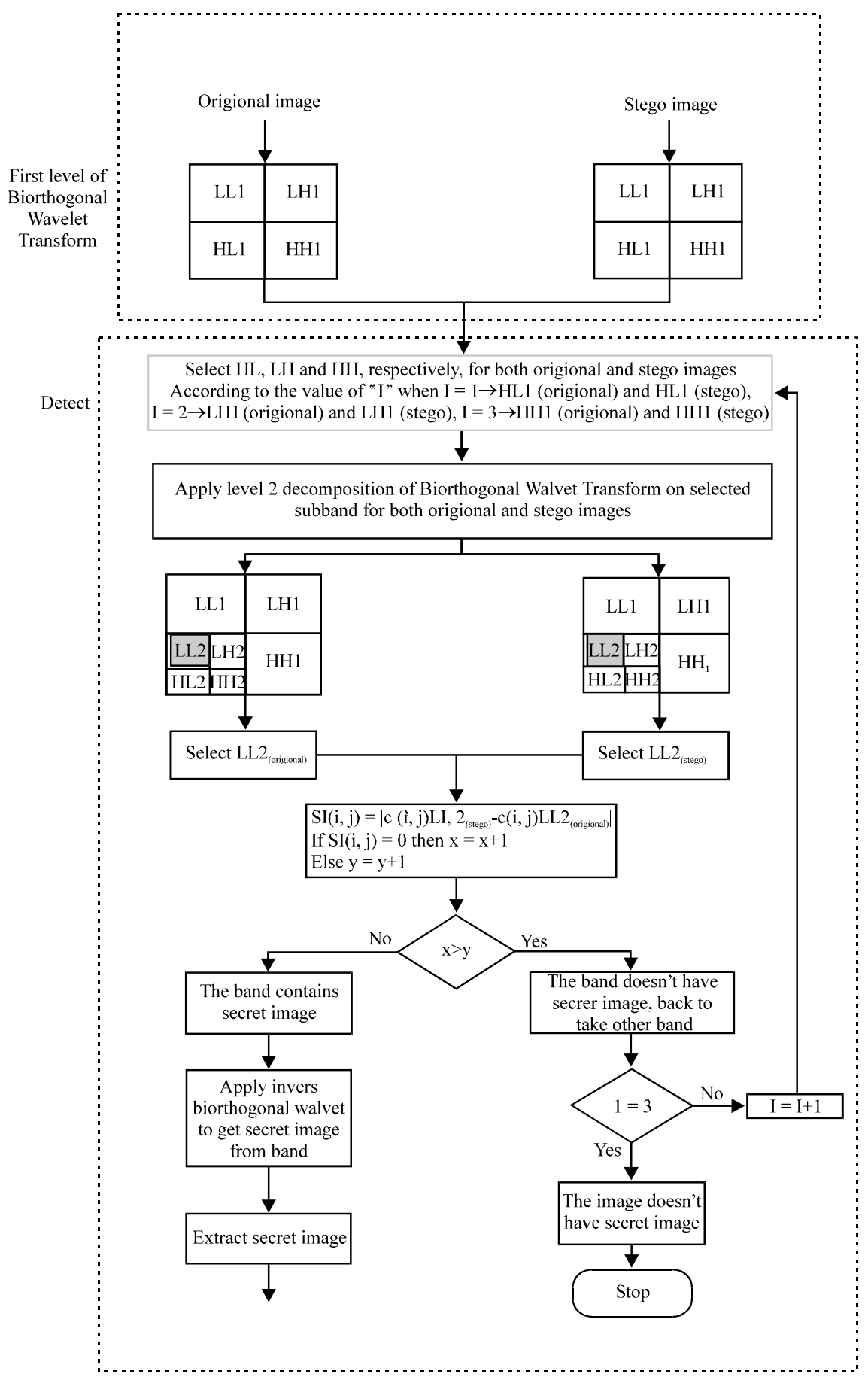

Fig. 6: Detect the sub-band of the extracting algorithm

image are subtracted from the coefficients for identical sub-band in stego-image as shown in Fig. 6. It is an appropriate way to detect the band that contains the secret image. This is because low frequency sub-band of any high frequency sub-bands have information equal to the secret image which means the secret image. While in first level of decomposition cannot possible to obtain clear results when subtracted the coefficients of high frequency sub-bands in original image from the coefficients in identical sub-band in stego-image. 


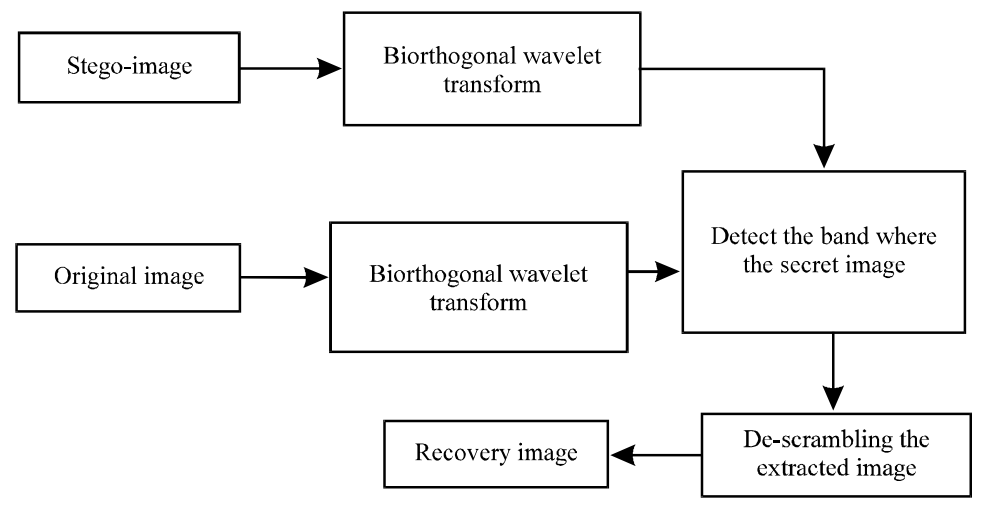

Fig. 7: General diagram of the extracting algorithm

\author{
Algorithm 2; The step of extraction algorithm: \\ Input: Stego-image \\ Output: Recover secret image \\ Step 1: Apply BWT to get the first level of decomposition $\left(\mathrm{LL}_{1}, \mathrm{LH}_{1}, \mathrm{HL}_{1}\right.$, \\ $\mathrm{HH}_{1}$ ) for both stego-image and original image, as in Fig. 7 \\ Step 2: Detect the band where the secret image by the following steps: \\ Step A: Select $\mathrm{HL}_{1}, \mathrm{LH}_{1}$ and $\mathrm{HH}_{1}$ sub-bands, respectively \\ Step B: Apply BWT on selected sub-band to get $\left(\mathrm{LL}_{2}, \mathrm{LH}_{2}, \mathrm{HL}_{2}, \mathrm{HH}_{2}\right)$ \\ sub-bands for both stego and original images \\ Step C: Select the $\mathrm{LL}_{2 \text { stego }}$ and $\mathrm{LL}_{\text {2original. }}$ \\ Step D: Subtract the coefficients of low frequency sub-bands by the \\ following equation: SI $(i, j)=\left|c^{\prime}(i, j)-c(i, j)\right|$ \\ where, the $c^{\prime}(i, j)$ represent the coefficients of $L_{2 \text { stego }}$ and $c(i, j)$ represent the \\ coefficients of $L_{2}$ original $S I(i, j)$ represent the pixel values of secret image. If \\ $\mathrm{x}>\mathrm{y}$ then the band doesn't have secret image where, $\mathrm{x}, \mathrm{y}$ are represent the \\ counters, back to Step (A) \\ Else secret image is contained in this band \\ Step E: Apply inverse BWT \\ Step F: Extract the scrambled secret image by divided it by $(\bullet)$ \\ Step 3: De-scramble the extracted image \\ Step 4: End
}

Verification measurements: In hiding techniques, one of the most important and most commonly used measurements to evaluate the performance is PSNR. It is a matrix used to measure the quality of the stego-image (Juneja and Sandhu, 2013). Typically, the range of PSNR values between 20-40 to good quality image (Salomon, 2007). It is measured as:

$$
\begin{gathered}
\text { PSNR }=10 \log _{10}\left(\frac{255^{2}}{\mathrm{MSE}}\right) \\
\mathrm{MSE}=\frac{1}{\mathrm{MN}} \sum_{\mathrm{x}=1}^{\mathrm{M}} \sum_{y=1}^{\mathrm{N}}\left(\mathrm{P}(\mathrm{x}, \mathrm{y})-\mathrm{P}^{\prime}(\mathrm{x}, \mathrm{y})\right)^{2}
\end{gathered}
$$

Where:

$\mathrm{M} \quad=$ Represents number of rows

$\mathrm{N}=$ The number of cover image columns

$\mathrm{P}(\mathrm{x}, \mathrm{y})$ and $=$ The Pixel values for the cover and $\mathrm{P}^{\prime}(\mathrm{x}, \mathrm{y}) \quad$ stego-image, respectively (Juneja and Sandhu, 2013)
The correlation measures the extent of similarity between the original secret image and the recovered image and can be calculated using the following Eq. 6 :

$$
\text { Corr }=\frac{\sum_{i=1}^{n}\left(x_{i}-\bar{x}\right)\left(y_{i}-\bar{y}\right)}{\sqrt{\sum_{i=1}^{n}\left(x_{i}-\bar{x}\right)^{2} \sum_{i=1}^{n}\left(y_{i}-\bar{y}\right)^{2}}}
$$

where, $x, y$ represents the secret image and recovered, respectively. Correlation of about 0.7 or above is counted passable (Pan et al., 2004). The maximum value of correlation is 1 (Swain and Lenka, 2014). Correlation is a standard for evaluating the robustness of data hiding technique while PSNR is the standard for assessing imperceptibility in it (Kasana et al., 2017).

\section{RESULTS AND DISCUSSION}

The proposed method was implemented in MATLAB 7.10.1. A set of test images were used to evaluate the results. The gray scale image of size $256 \times 256$ are used as cover images and $128 \times 128$ as secret images, Fig. 8 shows the test cover and secret images. In this research, the efficiency of Biorthogonal Wavelet 6.8 Transform in hiding process was studied. This algorithm invests the high frequency sub-bands $(\mathrm{HL}, \mathrm{LH}, \mathrm{HH})$ in the hiding process. To evaluate the efficiency of the hiding algorithm and to demonstrate the best hiding sub-band, PSNR and correlation criteria are used for test images. Table 1 displays the PSNR values of stego-images in different detail sub-bands.

Table 1, it is clear that the highest values of PSNR are obtained from the hiding in $\mathrm{HH}$ sub-band and the lowest values at $\mathrm{LH}$ and $\mathrm{HL}$ sub-bands, respectively. This is because of the presence of high 


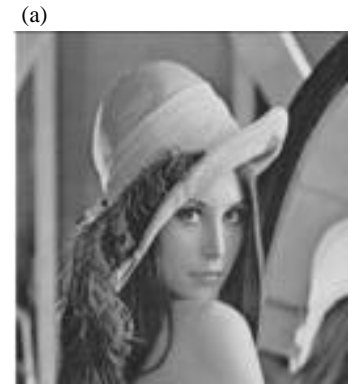

Lena

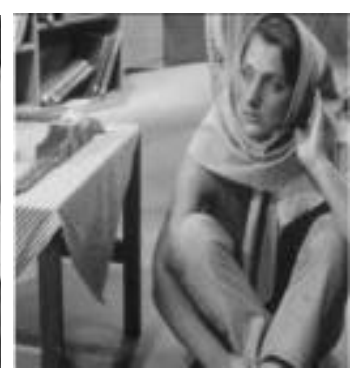

Barbara

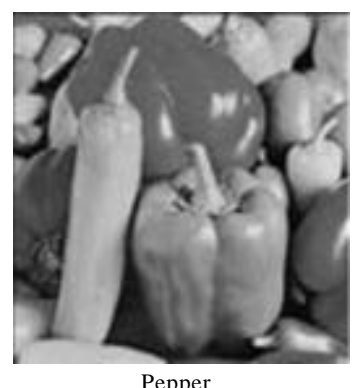

Pepper

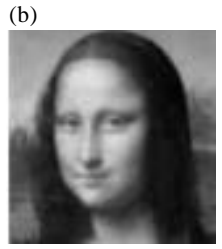

Monalisa

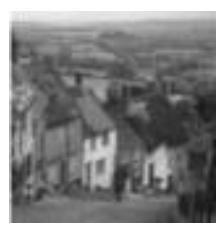

Goldhill

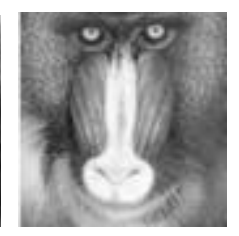

Baboon

Fig. 8: Test image of: a) Cover image and b) Secret image

frequencies in this sub-band, so, less distortion is obtained in the stego-images. While in $\mathrm{LH}$ and $\mathrm{HL}$ sub-bands the PSNR values are lower because of the existence of the low frequency. So, good imperceptibility can be obtained in using $\mathrm{HH}$ sub-band for hiding process.

A massive study has been done for Biorthogonal wavelet families. Table 2 shows the PSNR, Corr. values of stego-image for different biorthogonal and reverse biorthogonal wavelet transforms. Bior6.8 and rbio6.8 shows best results among other families which means best performance for both imperceptibility and robustness where the PSNR and Corr. values are highest. The stego-image obtained using rbio6.8 has a PSNR value higher than the value obtained using bior6.8 by small values when hiding in $\mathrm{HH}$ sub-band. The average PSNR of bior6.8 and rbio6.8 for $\mathrm{HH}$ sub-bands with various test images are found to be 35.7637 and $35.8133 \mathrm{~dB}$, respectively. On the other hand, the correlation for bior6.8 and rbio6.8 has acceptable values that are above $(0.7)$.

In $\mathrm{HH}$ sub-band for "Peppers" and "Baboon" test images, the reverse biorthogonal wavelet transforms (rbiol 5, rbio2.4, rbio3.5) used in the proposed method have higher PSNR values but Corr. is $<0.7$. This comparison shows that performance of the bior6.8 and rbio6.8 in terms of correlation is better than other families. The hiding algorithm have been compared with Haar wavelet transform based algorithm to show the effectiveness of biorthogonal wavelet transform over wavelet transform. Table 3 shows the results od PSNR and Corr. for the test stego-images. The stego-images obtained using bior6.8 and rbio6.8 have a PSNR value in hiding higher than the value obtained using by Haar wavelet at different sub-bands. For the Corr. values, it can be noted that the results of bior6.8 and rbio6.8 are accepted values while the Corr.values in Haar wavelet are high and always at the maximum value. Therefore, the algorithm of hiding by using bior 6.8 and rbio6. 8 provided good imperceptibility. Haar wavelet provided better results when compared in terms of (Corr.).

Table 4 shows the capacity, PSNR and Corr. of the proposed method in hiding at $\mathrm{HH}$ sub-band for bior6.8, rbio6.8 and Haar wavelet for Lena test image as host with different capacity of secret images. The experimental results shown that the proposed method has good performance with large data embedding capacity.

To illustrate the robustness of the proposed method versus noise attacks, in order to test the performance, the stego-image suffers some noise attacks when Lena test image as host with Monalisa of secret image. The Haar wavelet gives maximum value of correlation which equal to 1 , despite the exposure of the stego-image to the attacks. Whereas In bior6.8 and rbio6.8. When tested with Gaussian noise attack, maximum correlation between extracted and original secret data are $(0.7993$ and 0.7885$)$ and minimum are $(0.7858$ and 0.7744$)$. When tested with salt and pepper noise attack, maximum correlation between extracted and original secret data are $(0.7684$ and $0.7527)$ and minimum are $(0.7507$ and 0.7221$)$. From that one can observe that correlation between original and extracted secret data is close to one which means there is 
Table 1: The stego-images of BWT 6.8 for hiding process

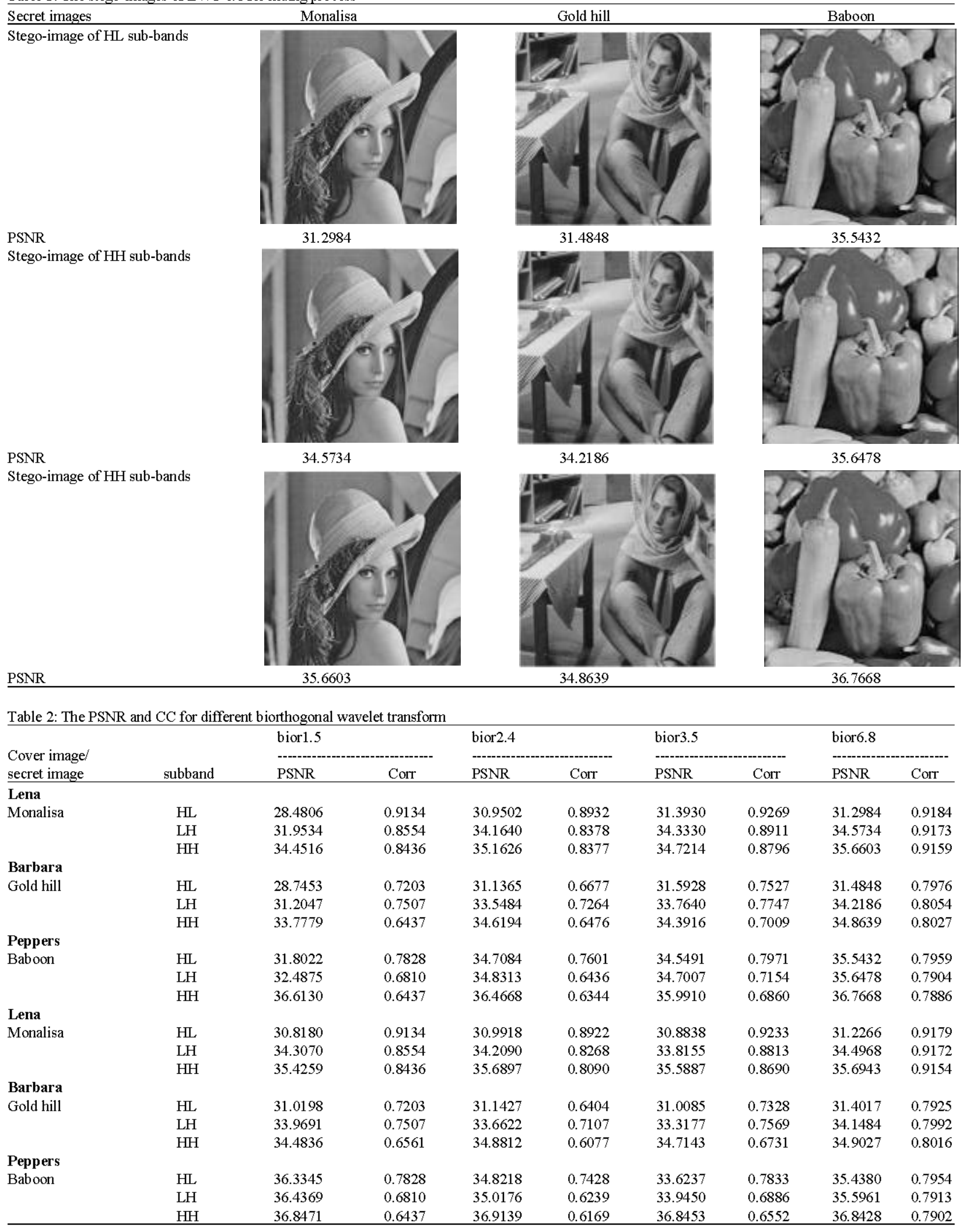


J. Eng. Applied Sci., 14 (Special Issue 6): 9396-9404, 2019

Table 3: The comparison between bior6.8, rbio6.8 and Haar wavelet transform

\begin{tabular}{|c|c|c|c|c|c|c|c|}
\hline \multirow{2}{*}{$\begin{array}{l}\text { Cover image/ } \\
\text { secret image }\end{array}$} & \multirow[b]{2}{*}{ subband } & \multicolumn{2}{|l|}{ Haar } & \multicolumn{2}{|l|}{ bior6.8 } & \multicolumn{2}{|l|}{ rbio6.8 } \\
\hline & & PSNR & Corr & PSNR & Corr & PSNR & Corr \\
\hline \multicolumn{8}{|l|}{ Lena } \\
\hline \multirow[t]{3}{*}{ Monalisa } & $\mathrm{HL}$ & 29.0740 & 1 & 31.2984 & 0.9184 & 31.2266 & 0.9179 \\
\hline & $\mathrm{LH}$ & 32.5873 & 1 & 34.5734 & 0.9173 & 34.4968 & 0.9172 \\
\hline & $\mathrm{HH}$ & 34.9013 & 1 & 35.6603 & 0.9159 & 35.6943 & 0.9154 \\
\hline \multicolumn{8}{|l|}{ Barbara } \\
\hline \multirow[t]{3}{*}{ Gold hill } & $\mathrm{HL}$ & 29.3400 & 1 & 31.4848 & 0.7976 & 31.4017 & 0.7925 \\
\hline & LH & 31.7220 & 1 & 34.2186 & 0.8054 & 34.1484 & 0.7992 \\
\hline & $\mathrm{HH}$ & 34.0999 & 1 & 34.8639 & 0.8027 & 34.9027 & 0.8016 \\
\hline \multicolumn{8}{|l|}{ Peppers } \\
\hline \multirow[t]{3}{*}{ Baboon } & $\mathrm{HL}$ & 32.0672 & 1 & 35.5432 & 0.7959 & 35.4380 & 0.7954 \\
\hline & $\mathrm{LH}$ & 32.7713 & 1 & 35.6478 & 0.7904 & 35.5961 & 0.7913 \\
\hline & $\mathrm{HH}$ & 36.6479 & 1 & 36.7668 & 0.7886 & 36.8428 & 0.7902 \\
\hline
\end{tabular}

Table 4: The capacity, PSNR, correlation from Lena stego-image

\begin{tabular}{|c|c|c|c|c|c|c|}
\hline \multirow[b]{2}{*}{ Capacity of secret image in bytes } & \multicolumn{2}{|l|}{ bior6.8 } & \multicolumn{2}{|l|}{ rbio6.8 } & \multicolumn{2}{|c|}{ Haar wavelet } \\
\hline & PSNR & Corr. & PSNR & Corr. & PSNR & Corr. \\
\hline 2447.36 & 35.6603 & 0.9159 & 35.6943 & 0.9154 & 34.9013 & 1 \\
\hline 3379.2 & 35.5985 & 0.8203 & 35.6436 & 0.8197 & 34.7258 & 1 \\
\hline 3584 & 35.5479 & 0.8019 & 35.5610 & 0.8019 & 34.6907 & 1 \\
\hline$\underline{4147.2}$ & 34.6717 & 0.7858 & 34.6878 & 0.7863 & 33.9575 & 1 \\
\hline
\end{tabular}

Table 5: The PSNR and Corr. of the noise attacks for bior6.8, rbio6.8 and Haar wavelet

\begin{tabular}{|c|c|c|c|c|c|c|}
\hline \multirow[b]{2}{*}{ Noise attacks/subbands } & \multicolumn{2}{|l|}{ bior6.8 } & \multicolumn{2}{|l|}{ rbio6.8 } & \multicolumn{2}{|c|}{ Haar wavelet } \\
\hline & PSNR & Corr. & PSNR & Corr. & PSNR & Corr. \\
\hline \multicolumn{7}{|c|}{ Gaussian noise mean $=0$, variance $=0.00005$} \\
\hline $\mathrm{HL}$ & 31.0085 & 0.7858 & 30.9416 & 0.7845 & 28.9008 & 1 \\
\hline LH & 33.9785 & 0.7892 & 33.9132 & 0.7885 & 32.1920 & 1 \\
\hline $\mathrm{HH}$ & 34.9097 & 0.7993 & 34.9335 & 0.7744 & 34.2624 & 1 \\
\hline \multicolumn{7}{|c|}{ Salt and pepper noise (0.01) } \\
\hline $\mathrm{HL}$ & 27.2619 & 0.7519 & 27.2340 & 0.7527 & 23.7965 & 1 \\
\hline LH & 28.2734 & 0.7507 & 28.2559 & 0.7523 & 24.5654 & 1 \\
\hline $\mathrm{HH}$ & 28.5042 & 0.7684 & 28.5102 & 0.7221 & 24.8537 & 1 \\
\hline \multicolumn{7}{|l|}{ Speckle noise $(0.001)$} \\
\hline $\mathrm{HL}$ & 29.9172 & 0.5509 & 29.8660 & 0.5526 & 28.2284 & 1 \\
\hline LH & 32.0393 & 0.5515 & 31.9972 & 0.5525 & 30.8334 & 1 \\
\hline $\mathrm{HH}$ & 32.6139 & 0.5862 & 32.6322 & 0.5421 & 32.2404 & 1 \\
\hline
\end{tabular}

very less degradation in secret data. While in the speckle noise attack the Corr. values are lowest but the experimental results show that the values of Corr. increased from 0.5-0.7 when $\cdot=0.1$, the Corr. values becomes (0.7692 and 0.7499) and the PSNR values are found to be (30.1902 and 30.2502) from hiding in $\mathrm{HH}$ sub-bands. Table 5 shows the noise attacks on different sub-bands at $\bullet=0.05$.

\section{CONCLUSION}

This study propose steganography system which based on biorthogonal wavelet transform. The biorthogonal wavelet transform provides good capability for both the stego-image and secret image. This makes the hiding method efficient and invisible. This algorithm can hide the secret image in any one of high frequency sub-bands. Before hiding the secret image in the cover, scrambling process is applied to the positions of the rows and then columns which would give strength to the hiding process. So, the secret image is difficult identify for someone he wants to attack. The experimental results show that the best results are obtained when hiding in $\mathrm{HH}$. The comparison of biorthogonal reverse Biorthogonal wavelet families and Haar wavelet transform is done through the imperceptibility measure (PSNR) and robustness measure (Corr.). It is found that bior6.8 and rbio6.8 are better than other wavelets and provide better performance in terms of PSNR. The Corr. values are considered acceptance which close to one that means there is very less degradation in secret data.

\section{REFERENCES}

Akhtar, J., 2001. Optimization of biorthogonal wavelet filters for signal and image compression. Masters Thesis, Candidatus Scientarium at the Department of Informatics, University of Oslo, Oslo, Norway. 
Arvind, H.S. and N. Shankar, 2013. Reversible watermarking based on bi-orthogonal wavelet. J. Eng. Sci. Invention, 2: 76-85.

Atawneh, S. and P. Sumari, 2015. An overview of frequency-based digital image steganography. Intl. J. Cryptology Res., 5: 15-27.

Cheddad, A., J. Condell, K. Curran and P. McKevitt, 2008. Enhancing steganography in digital images. Proceedings of the Canadian Conference on Computer and Robot Vision, May 28-30, 2008, Windsor, ON., Canada, pp: 326-332.

Divya, A. and H.K. Priya, 2013. A wavelet transform based watermarking algorithm for protecting copyrights of digital images. ICTACT. J. Image Video Process., 4: 657-660.

Houssein, E.H., M.A. Ali and A.E. Hassanien, 2016. An image Steganography algorithm using Haar discrete wavelet transform with advanced encryption system. Proceedings of the 2016 International Federated Conference on Computer Science and Information Systems (FedCSIS), September 11-14, 2016, IEEE, Gdansk, Poland, ISBN:978-1-5090-0046-3, pp: 641-644.

Jiansheng, M., L. Sukang and T. Xiaomei, 2009. A digital watermarking algorithm based on DCT and DWT. Proceedings of the International Symposium on Web Information Systems and Applications, May 22-24, 2009, Academy Publisher, Guwahati, India, ISBN:978-952-5726-00-8, pp: 104-107.

Juneja, M. and P.S. Sandhu, 2013. A new approach for information security using an improved steganography technique. J. Inf. Process. Syst., 9: 405-424.

Kasana, G., K. Singh and S.S. Bhatia, 2017. Data hiding algorithm for images using discrete wavelet transform and arnold transform. J. Inf. Process. Syst., 13: 1331-1344.
Kumar, S. and S.K. Muttoo, 2013. Image steganography based on wavelet families. J. Comput. Eng. Inf. Technol., 2: 1-9.

Nag, A., S. Biswas, D. Sarkar and P.P. Sarkar, 2011. A novel technique for image steganography based on DWT and Huffman encoding. Int. J. Comput. Sci. Inform. Technol., 4: 561-570.

Pan, J.S., H.C. Huang and L.C. Jain, 2004. Intelligent Watermarking Techniques. Vol. 7, World scientific, Singapore, ISBN:9789812387578, Pages: 852.

Reddy, M.I.S. and A.S. Kumar, 2016. Secured data transmission using wavelet based steganography and cryptography by using AES algorithm. Procedia Comput. Sci., 85: 62-69.

Salomon, D., 2007. Data Compression: The Complete Reference. 4th Edn., Springer, Berlin, Germany, ISBN:9781846286032, Pages: 1092.

Singh, P. and R.S. Chadha, 2013. A survey of digital watermarking techniques, applications and attacks. Intl. J. Eng. Innovative Technol., 2: 165-175.

Stolojescu, C.L., 2011. A wavelets based approach for time series mining. Ph.D Thesis, West University of Timisoara Timisoara, Romania.

Swain, G. and S.K. Lenka, 2014. Classification of image steganography techniques in spatial domain: A study. Intl. J. Comput. Sci. Eng. Technol., 5: 219-232.

Toufik, B. and N. Mokhtar, 2012. The Wavelet Transform for Image Processing Applications. In: Advances in Wavelet Theory and Their Applications in Engineering, Physics and Technology, Baleanu, D. and H. Aydin (Eds.). IntechOpen Limited, London, UK., ISBN:9789535104940, pp: 1-31.

Wavelets, 2018. Biorthogonal wavelet transform, Pywavelets. Filip Wasilewski, San Francisco, USA. http://wavelets.pybytes.com/family/bior/ 